\title{
INVARIANT MEASURES FOR CHEBYSHEV MAPS ${ }^{1}$
}

\author{
ABRAHAM BOYARSKY \\ PAWEE GORA \\ Concordia University \\ Department of Mathematics and Statistics, 7141 Sherbrooke Street West \\ Montreal, Quebec H4B 1R6, Canada \\ E-mail: boyar@vax2.concordia.ca,pgora@vax2.concordia.ca
}

(Received April, 2000; Revised November, 2000)

Let $T_{\lambda}(x)=\cos (\lambda \arccos x),-1 \leq x \leq 1$, where $\lambda>1$ is not an integer. For a certain set of $\lambda$ 's which are irrational, the density of the unique absolutely continuous measure invariant under $T_{\lambda}$ is determined exactly. This is accomplished by showing that $T_{\lambda}$ is differentially conjugate to a piecewise linear Markov map whose unique invariant density can be computed as the unique left eigenvector of a matrix.

Key words: Chebyshev Map, Absolutely Continuous Invariant Measure, Markov Map.

AMS subject classifications: $37 \mathrm{~A} 05,37 \mathrm{E} 05$.

\section{Chebyshev Maps}

The function $T_{n}(x)=\cos (n \arccos x), \quad-1 \leq x \leq 1, n=0,1,2, \ldots$ defines the $n$th Chebyshev polynomial, which is a solution of the differential equation

$$
\left(1-x^{2}\right) y^{\prime \prime}-x y^{\prime}+n^{2} y=0
$$

Polynomial $T_{n}, n \geq 2$, transforms each of the intervals $\left[\frac{i}{n-1}, \frac{i+1}{n-1}\right], i=-(n-1), \ldots$, $(n-1)-1$, onto $[-1,1]$. It is easy to show (see [1]) that the unique absolutely continuous invariant measure for all $T_{n}$ 's is

$$
d \mu(x)=\frac{1}{\pi \sqrt{1-x^{2}}} d x .
$$

In this note, we consider the family of Chebyshev maps $T_{\lambda}(x)=\cos (\lambda \arccos x)$, $-1 \leq x \leq 1$, where $\lambda>1$ is not an integer. $T_{\lambda}$ is a solution of the same differential equation (1) with $n$ replaced by $\lambda$, but $T_{\lambda}$ is no longer a polynomial and we, therefore, refer to it as a Chebyshev map. The first monotonic branch of $T_{\lambda}$ is not onto, but all the others are (see Figure 1).

\footnotetext{
${ }^{1}$ The research of both authors was supported by NSERC grants.
} 


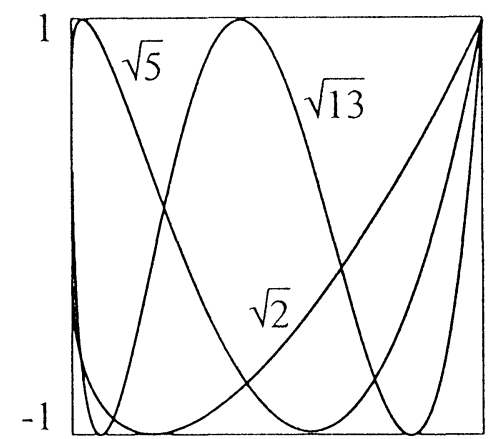

$-1$

Figure 1: Chebyshev maps for $\lambda=\sqrt{2}, \sqrt{5}, \sqrt{13}$.

The main result of this note characterizes a set of $\lambda$ 's, not integers, for which the unique absolutely continuous measure invariant under $T_{\lambda}$ can be determined exactly. This is accomplished by showing that $T_{\lambda}$ is differentially conjugate to a piecewise linear Markov map whose unique invariant density can be computed as the unique left eigenvector of a matrix.

A related problem of finding an absolutely continuous invariant measure for a piecewise linear map with two branches $(1<\lambda<2)$ was considered in [3].

\section{Differentiable Conjugacy}

For $\lambda>1$, let $\Lambda_{\lambda}:[0,1] \rightarrow[0,1]$ be a piecewise linear continuous map having slope $\mp \lambda$ and defined by joining points $(0,0),\left(\frac{1}{\lambda}, 1\right),\left(\frac{2}{\lambda}, 0\right),\left(\frac{3}{\lambda}, 1\right), \ldots$. Depending on $\lambda$, either of the two situations shown in Figure 2 can occur, where $[\lambda]$ is the largest integer less than or equal to $\lambda$.
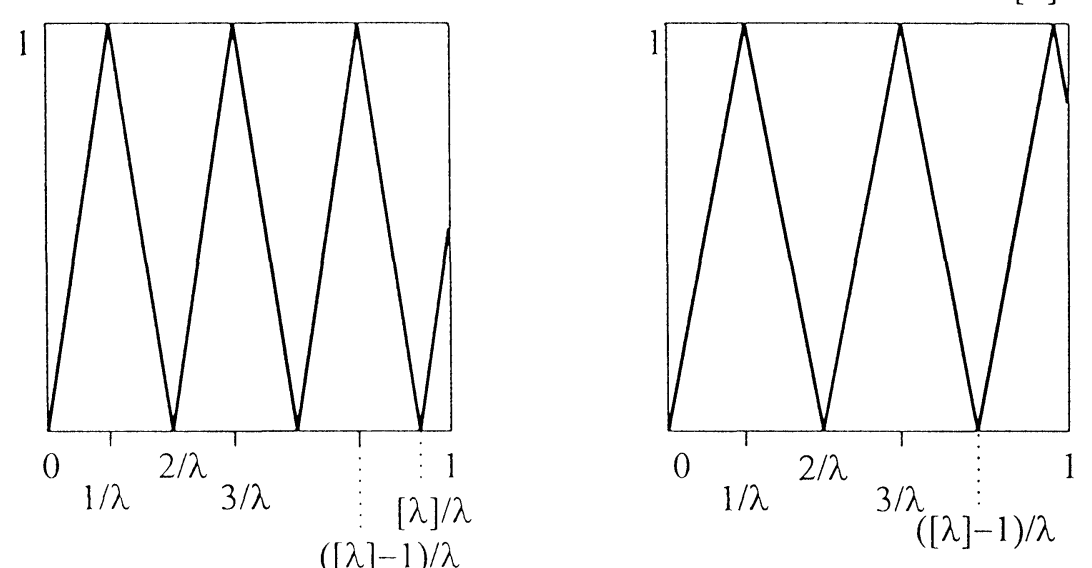

Figure 2: Two possible shapes of $\Lambda_{\lambda}$. 
Let $h:[0,1] \rightarrow[-1,1]$ be defined by $h(x)=\cos (\pi x)$. Both $h$ and $h^{-1}$ are continuous differentiable.

Proposition 1: For any $\lambda>1$, we have $\Lambda_{\lambda}=h^{-1} \circ T_{\lambda} \circ h$, i.e., $T_{\lambda}$ is differentially conjugated to the triangle map $\Lambda_{\lambda}$.

Proof: It is enough to show that $T_{\lambda} \circ h=h \circ \Lambda_{\lambda}$. Let us consider $x$ in the intervals $\left[\frac{k}{\lambda}, \frac{k+1}{\lambda}\right]$, for $0 \leq k \leq[\lambda]-1$ and $\left[\frac{[\lambda]}{\lambda}, 1\right]$, for $k=[\lambda]$. For $k$ even, we have $\Lambda_{\lambda}(x)=\lambda\left(x-\frac{k}{\lambda}\right)$. Thus

$$
h\left(\Lambda_{\lambda}(x)\right)=\cos (\pi \lambda x-\pi k)=\cos (\pi \lambda x) .
$$

For $k$ odd, we have $\Lambda_{\lambda}(x)=1-\lambda\left(x-\frac{k}{\lambda}\right)$. Therefore,

$$
h\left(\Lambda_{\lambda}(x)\right)=\cos (\pi-\pi \lambda x+\pi k)=\cos (\pi \lambda x) .
$$

On the other hand, we always have

$$
T_{\lambda}(h(x))=\cos (\lambda \arccos (\cos (\pi x)))=\cos (\lambda \pi x) .
$$

This proves the claimed conjugation.

Remark 1: For $[\lambda]$ even, we have $\Lambda_{\lambda}(1)=\lambda-[\lambda]$. For odd $[\lambda]$, we have $\Lambda_{\lambda}(1)=$ $1-(\lambda-[\lambda])$.

\section{The Measure Invariant Under a Chebyshev Map}

Definition: Let $\tau:[0,1] \rightarrow[0,1]$ be a piecewise monotonic map for which there exist points $0=a_{0}<a_{1}<\ldots<a_{n-1}<a_{n}=1$ such that for $i=0,1, \ldots, n-1,\left.\tau\right|_{\left(a_{i}, a_{i+1}\right)}$ is a homeomorphism onto some interval $\left(a_{j(i)}, a_{k(i)}\right)$. Then $\tau$ is called Markov with respect to the partition $\left(a_{1}, a_{2}, \ldots, a_{n}\right)$.

The main tool in exploring absolutely continuous invariant measures of $\tau$ is the Frobenius-Perron operator $P_{\tau}$ on the space of Lebesgue integrable functions $L^{1}([0,1])$ :

$$
\left(P_{\tau} f\right)(x)=\sum_{i=1}^{n} \frac{f\left(\tau_{i}^{-1}(x)\right)}{\left|\tau^{\prime}\left(\tau_{i}^{-1}(x)\right)\right|}
$$

where $\tau_{i}^{-1}, i=1, \ldots, n$, are inverse branches of $\tau$. A function $f$ satisfies $P_{\tau} f=f$ if and only if $f$ is the density of an absolutely continuous $\tau$-invariant measure. For more detailed information on $P_{\tau}$, see [1].

Let $\mathscr{P}$ be the partition $\left(0, \frac{1}{\lambda}, \frac{2}{\lambda}, \ldots, \frac{[\lambda]}{\lambda}, 1\right)$. Clearly, if $\lambda$ is a positive integer, $\Lambda_{\lambda}$ is a Markov map with respect to $\mathscr{9}$. Below we characterize the set of $\lambda$ 's, not integers, for which $\Lambda_{\lambda}$ is a Markov map with respect to $\mathscr{\rho}$. It is easy to see that $\Lambda_{\lambda}$ is Markov if and only if $\Lambda_{\lambda}(1)=\frac{m}{\lambda}$, where $m$ is an integer satisfying $1 \leq m \leq[\lambda]$.

Proposition 2: If $\lambda$ is not an integer, then $\Lambda_{\lambda}$ is Markov with respect to of if and only if:

Case 1:

$$
\lambda=n+\sqrt{n^{2}+m}, \text { for }[\lambda]=2 n \text { and } 1 \leq m \leq 2 n ;
$$




\section{Case 2:}

$$
\lambda=(n+1)+\sqrt{(n+1)^{2}-m}, \text { for }[\lambda]=2 n+1 \text { and } 1 \leq m \leq 2 n .
$$

In both cases, $\lambda$ is irrational. In (3), $m$ cannot be equal to $2 n+1$ since then $\lambda$ would be an integer.

Proof: We use the values of $\Lambda_{\lambda}(1)$ from Remark 1. In Case 1, we have $\Lambda_{\lambda}(1)=\lambda-2 n=\frac{m}{\lambda}$, or $\lambda^{2}-2 \lambda n-m=0$, whose positive solution is given in (2). In Case 2 , we have $\Lambda_{\lambda}(1)=1-(\lambda-2 n-1)=\frac{m}{\lambda}$, or $\lambda^{2}-2 \lambda(n+1)+m=0$. The only positive solution is given in (3).

Remark 2: There are no non-integer solutions to $(3)$ for $[\lambda]=1$. Thus, the smallest $[\lambda]$ we can actually consider is $[\lambda]=2$.

When $\Lambda_{\lambda}$ is Markov with respect to $\mathscr{\rho}$, then its Frobenius-Perron operator restricted to the piecewise constant functions on the partition $\mathscr{P}$, can be represented by the $\lambda_{1} \times \lambda_{1}$ matrix $\mathbb{M}$, where $\lambda_{1}=[\lambda]+1$. For $[\lambda]=2 n$ and $\lambda-[\lambda]=\frac{m}{\lambda}$ (Case 1 ), we have

$$
\mathbb{M}=\left[\begin{array}{ccccccc}
\frac{1}{\lambda} & \frac{1}{\lambda} & \ldots & \ldots & \ldots & \frac{1}{\lambda} & \frac{1}{\lambda} \\
\frac{1}{\lambda} & \frac{1}{\lambda} & \ldots & \ldots & \ldots & \frac{1}{\lambda} & \frac{1}{\lambda} \\
\vdots & \vdots & \ldots & \ldots & \ldots & \vdots & \vdots \\
\frac{1}{\lambda} & \frac{1}{\lambda} & \ldots & \ldots & \ldots & \frac{1}{\lambda} & \frac{1}{\lambda} \\
\frac{1}{\lambda} & \frac{1}{\lambda} & \ldots & \frac{1}{\lambda} & 0 & \ldots & 0
\end{array}\right]
$$

where the upper $\lambda_{1}-1$ rows consists of $\frac{1}{\lambda}$ 's and there are exactly $m \frac{1}{\lambda}$ 's at the beginning of the last row. If $[\lambda]=2 n+1$ and $1-(\lambda-[\lambda])=\frac{m}{\lambda}$ (Case 2), the matrix $M$ is similar, except that the last row starts with $m$ zeros and ends with $\left(\lambda_{1}-m\right) \frac{1}{\lambda}$ 's.

Let $f=\left(f_{1}, f_{2}, \ldots, f_{\lambda_{1}}\right)$ represent a piecewise constant function on the partition $\left(0, \frac{1}{\lambda}, \frac{2}{\lambda}, \ldots, \frac{[\lambda]}{\lambda}, 1\right)$. We consider the equation $f \mathbb{M}=f$ with the normalizing condition which makes $f$ a density of a probability measure:

$$
\sum_{i=1}^{[\lambda]} f_{i} \frac{1}{\lambda}+f_{\lambda_{1}}\left(1-\frac{[\lambda]}{\lambda}\right)=1
$$

Case 1: $[\lambda]=2 n$ and $\lambda-[\lambda]=\frac{m}{\lambda}$. Then, $f \mathbb{M}=f$ reduces to

$$
\begin{gathered}
f_{1}=f_{2}=\ldots=f_{m} \\
f_{m+1}=f_{m+2}=\ldots=f_{\lambda_{1}} \\
f_{1}=\frac{m}{\lambda} f_{1}+\frac{\lambda_{1}-m}{\lambda} f_{\lambda_{1}}
\end{gathered}
$$




$$
f_{\lambda_{1}}=\frac{m}{\lambda} f_{1}+\frac{\lambda_{1}-1-m}{\lambda} f_{\lambda_{1}}
$$

Since $\lambda=[\lambda]=\frac{m}{\lambda}$, the last two equations coincide and we obtain:

$$
f_{1}=\frac{\lambda_{1}-m}{\lambda-m} f_{\lambda_{1}}
$$

Substituting into (4), we get

$$
\frac{m}{\lambda} \frac{\lambda_{1}-m}{\lambda-m} f_{\lambda_{1}}+\frac{[\lambda]-m}{\lambda} f_{\lambda_{1}}+\left(1-\frac{[\lambda]}{\lambda}\right) f_{\lambda_{1}}=1
$$

which gives

$$
f_{\lambda_{1}}=\frac{\lambda(\lambda-m)}{m\left(\lambda_{1}-m\right)+(\lambda-m)^{2}} \text { and } f_{1}=\frac{\lambda\left(\lambda_{1}-m\right)}{m\left(\lambda_{1}-m\right)+(\lambda-m)^{2}}
$$

Case 2: $[\lambda]=2 n+1$ and $1-(\lambda-[\lambda])=\frac{m}{\lambda}$. Considerations analogous to that of Case 1 lead to:

$$
f_{\lambda_{1}}=\frac{\lambda(\lambda-m)}{m([\lambda]-m)+(\lambda-m)^{2}} \text { and } f_{1}=\frac{\lambda([\lambda]-m)}{m([\lambda]-m)+(\lambda-m)^{2}} .
$$

We have proved the following theorem.

Theorem 1: If $\lambda>1$ is such that $\Lambda_{\lambda}$ is a Markov map with respect to $\mathcal{P}$, then the unique invariant density of $\Lambda_{\lambda}$ is: for $[\lambda]=2 n$

and for $[\lambda]=2 n+1$ :

$$
f_{\lambda}(x)= \begin{cases}\frac{\lambda\left(\lambda_{1}-m\right)}{m\left(\lambda_{1}-m\right)+(\lambda-m)^{2}} & \text { for } 0 \leq x<\frac{m}{\lambda} \\ \frac{\lambda(\lambda-m)}{m\left(\lambda_{1}-m\right)+(\lambda-m)^{2}} & \text { for } \frac{m}{\lambda} \leq x \leq 1 ;\end{cases}
$$

$$
f_{\lambda}(x)= \begin{cases}\frac{\lambda([\lambda]-m)}{m([\lambda]-m)+(\lambda-m)^{2}} & \text { for } 0 \leq x<\frac{m}{\lambda} \\ \frac{\lambda(\lambda-m)}{m([\lambda]-m)+(\lambda-m)^{2}} & \text { for } \frac{m}{\lambda} \leq x \leq 1 .\end{cases}
$$

Now, we can use the differentiable conjugacy $h$ of Proposition 1 to find invariant densities for Markov Chebyshev maps. By Proposition 2 of [2], the $T_{\lambda}$-invariant density is

$$
F_{\lambda}(x)=f_{\lambda}\left(h^{-1}(x)\right)\left|\left(h^{-1}\right)^{\prime}\right|=f_{\lambda}\left(\frac{1}{\pi} \arccos x\right) \frac{1}{\pi \sqrt{1-x^{2}}} .
$$

Thus the following theorem holds.

Theorem 2: If $\lambda>1$ is such that $T_{\lambda}$ is a Markov map with respect to 9 , then the unique invariant density of $T_{\lambda}$ is 


$$
F_{\lambda}(x)= \begin{cases}\frac{f_{1}}{\pi \sqrt{1-x^{2}}}, & \text { for } 0 \leq \frac{1}{\pi} \arccos x<\frac{m}{\lambda} \\ \frac{f_{\lambda_{1}}}{\pi \sqrt{1-x^{2}}}, & \text { for } \frac{m}{\lambda} \leq \frac{1}{\pi} \arccos x \leq 1\end{cases}
$$

where $m$ satisfies $\lambda-[\lambda]=\frac{m}{\lambda}$ for $[\lambda]=2 n$ or $1-(\lambda-[\lambda])=\frac{m}{\lambda}$ for $[\lambda]=2 n+1$ and constants $f_{1}, f_{\lambda_{1}}$ are given by formulas (5) or (6), respectively.

Remark 3: For a non-integer $\lambda$, we always have $\left|T_{\lambda}^{\prime}(-1+t)\right|=O\left((\sqrt{1-t})^{-1}\right)$, as $t \rightarrow 0^{+}$, which explains the lack of singularity of $F_{\lambda}$ at $\frac{m}{\lambda}$.

\section{Examples}

Example 1: Using Maple V, release 5, we have calculated some values of $\lambda$ and corresponding values of $f_{1}$ and $f_{\lambda_{1}}$. They are presented in the tables below.

Case 1:

\begin{tabular}{|c|c|c|c|}
$(n, m)$ & $\lambda$ & $f_{1}$ & $f_{\lambda_{1}}$ \\
\hline$(1,1)$ & 2.414213562 & 1.207106781 & .8535533903 \\
\hline$(5,7)$ & 10.65685425 & 1.030330086 & .9419417381 \\
\hline$(100,107)$ & 200.5335765 & 1.002319740 & .9973462776 \\
\hline
\end{tabular}

\section{Case 2:}

\begin{tabular}{|c|c|c|c|}
$(n, m)$ & $\lambda$ & $f_{1}$ & $f_{\lambda_{1}}$ \\
\hline$(1,1)$ & 3.732050808 & .7886751346 & 1.077350270 \\
\hline$(5,7)$ & 11.38516481 & .9642383460 & 1.057086015 \\
\hline$(100,107)$ & 201.4689006 & .9976664394 & 1.002643103 \\
\hline
\end{tabular}

For some $\lambda>1$, the map $\Lambda_{\lambda}$ may not be Markov with respect to the partition o but will be Markov with respect to some finer partition. In all such cases, it is possible to find an invariant density of $\Lambda_{\lambda}$ and then use it to find the invariant density of the Chebyshev map $T_{\lambda}$. Below, we present two simple examples of such situations.

Example 2: Let us look for $\lambda$ such that $[\lambda]=1$ and $\Lambda_{\lambda}(1)=\frac{1}{\lambda^{2}}$, see Figure 3a). Then $\lambda\left(1-\frac{1}{\lambda}\right)=1-\frac{1}{\lambda^{2}}$, or $\lambda^{3}-2 \lambda^{2}+1=0$. The non-integer positive solution is $\lambda=\frac{1}{2}(1+\sqrt{5}) \approx 1.618$. It is easy to see that $\Lambda_{\lambda}$ is Markov with respect to the partition $\mathbb{Q}=\left(0, \frac{1}{\lambda^{2}}, \frac{1}{\lambda}, 1\right)$ and the corresponding Frobenius-Perron matrix is 


$$
\mathbb{M}=\left[\begin{array}{ccc}
\frac{1}{\lambda} & \frac{1}{\lambda} & 0 \\
0 & 0 & \frac{1}{\lambda} \\
0 & \frac{1}{\lambda} & \frac{1}{\lambda}
\end{array}\right]
$$

Its left invariant vector is $f=\left(f_{1}, f_{2}, f_{3}\right)=(0,1, \lambda) f_{2}$ and after the normalization the $\Lambda_{\lambda}$-invariant density is $f=\left(0, \frac{\lambda+1}{2 \lambda-1}, \frac{2 \lambda+1}{2 \lambda-2}\right) \approx(0,1.171,1.894)$.
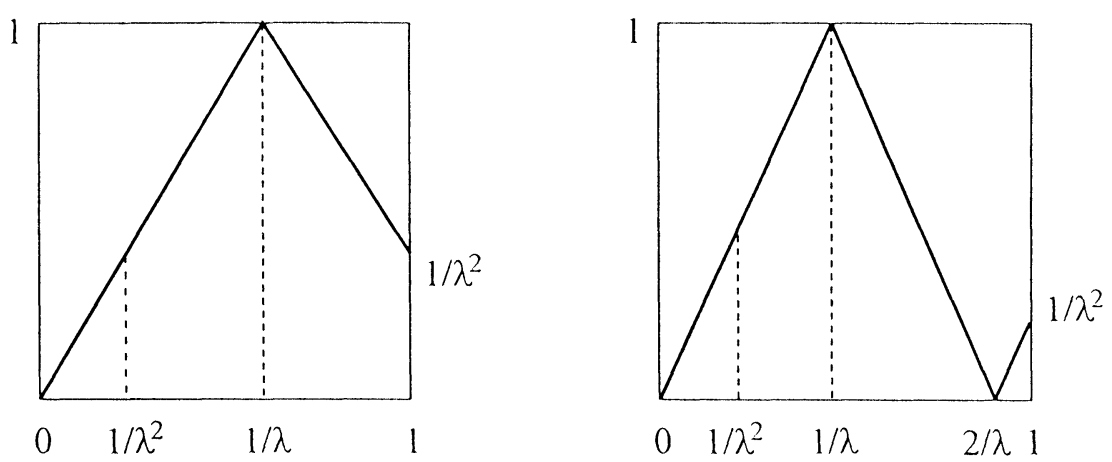

Figure 3

Example 3: Let us look for $\lambda$ such that $[\lambda]=2$ and $\Lambda_{\lambda}(1)=\frac{1}{2}$, see Figure $3 \mathrm{~b}$ ). Then, $\lambda\left(1-2 \frac{1}{\lambda}\right)=\frac{1}{\lambda^{2}}$, or $\lambda^{3}-2 \lambda^{2}-1=0$. The only real solution is $\lambda \approx 2.206$. It is easy to see that $\Lambda_{\lambda}$ is Markov with respect to the partition $\mathbb{Q}=\left(0, \frac{1}{\lambda^{2}}, \frac{1}{\lambda}, \frac{2}{\lambda}, 1\right)$. The corresponding Frobenius-Perron matrix is

$$
\mathbb{M}=\left[\begin{array}{cccc}
\frac{1}{\lambda} & \frac{1}{\lambda} & 0 & 0 \\
0 & 0 & \frac{1}{\lambda} & \frac{1}{\lambda} \\
\frac{1}{\lambda} & \frac{1}{\lambda} & \frac{1}{\lambda} & \frac{1}{\lambda} \\
\frac{1}{\lambda} & 0 & 0 & 0
\end{array}\right]
$$

Its left invariant vector is $f=\left(f_{1}, f_{2}, f_{3}, f_{4}\right)=\left(\lambda^{2}-\lambda-1, \lambda-1,1,1\right) f_{3}$ and after normalization, the $\Lambda_{\lambda^{-}}$invariant density is $f=\frac{1}{3 \lambda-4}\left(\lambda^{2}-\lambda+1, \lambda^{2}-\lambda, \lambda, \lambda\right) \approx(1.398$, $1.016, .843, .843)$.

\section{Acknowledgement}

The authors are grateful to M. Scarowsky for helpful comments. 


\section{References}

[1] Boyarsky, A. and Góra, P., Laws of Chaos: Invariant Measures and Dynamical Systems in One Dimension, Birhaüser, New York 1997.

[2] Boyarsky, A. and Scarowsky, M., On a class of transformations which have unique absolutely continuous invariant measures, Trans. AMS 255 (1979), 243-262.

[3] Derrida, B., Gervois, A. and Pomeau, Y., Iteration of endomorphisms on the real axis and representation of numbers, Ann. Inst. H. Poincaré Sect. A (N.S.) 29 (1978), 305-356.

[4] Lasota, A. and Yorke, J.A., On the existence of invariant measures for piecewise monotonic transformations, Trans. AMS 186 (1973), 481-488. 


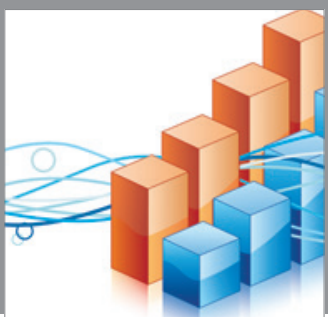

Advances in

Operations Research

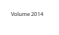

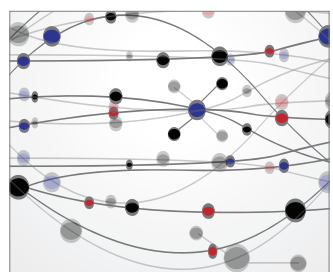

\section{The Scientific} World Journal
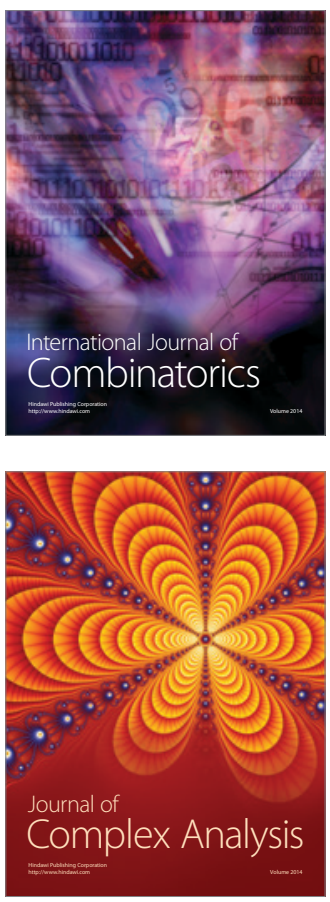

International Journal of

Mathematics and

Mathematical

Sciences
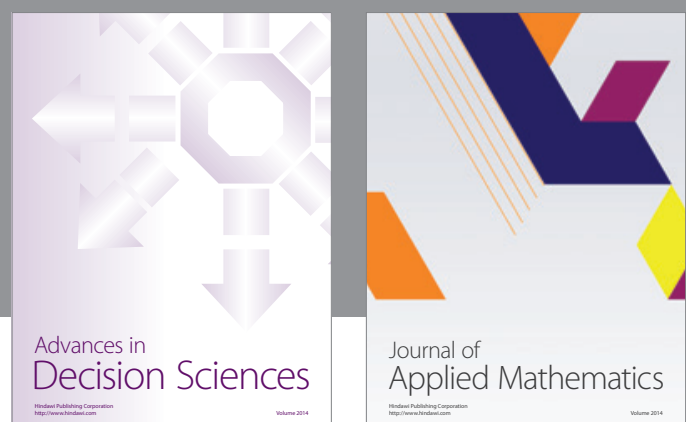

Journal of

Applied Mathematics
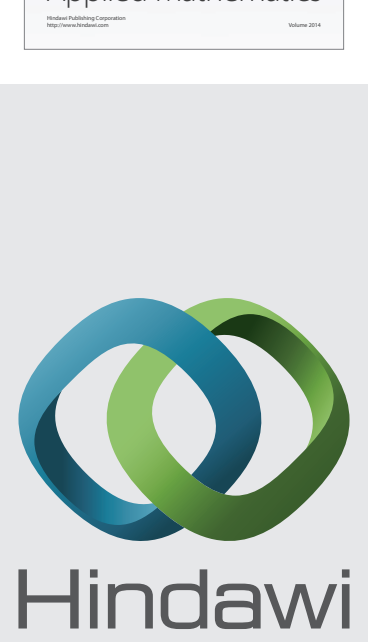

Submit your manuscripts at http://www.hindawi.com
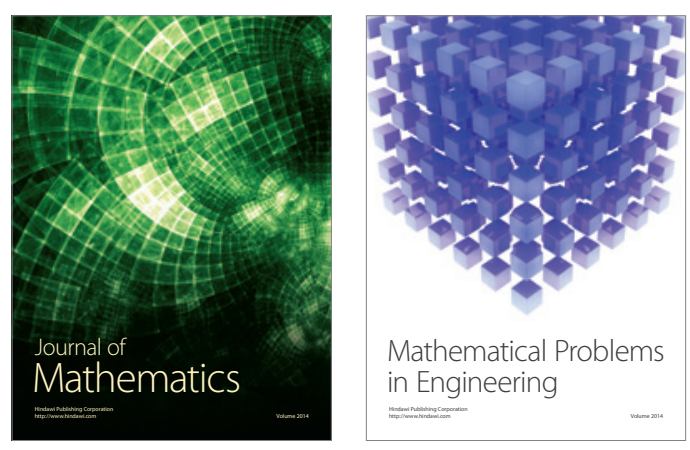

Mathematical Problems in Engineering
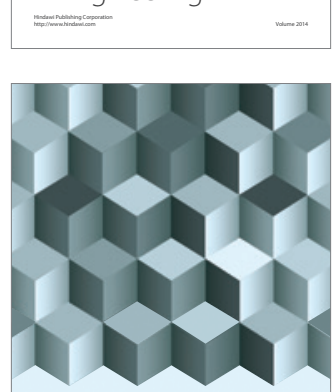

Journal of

Function Spaces
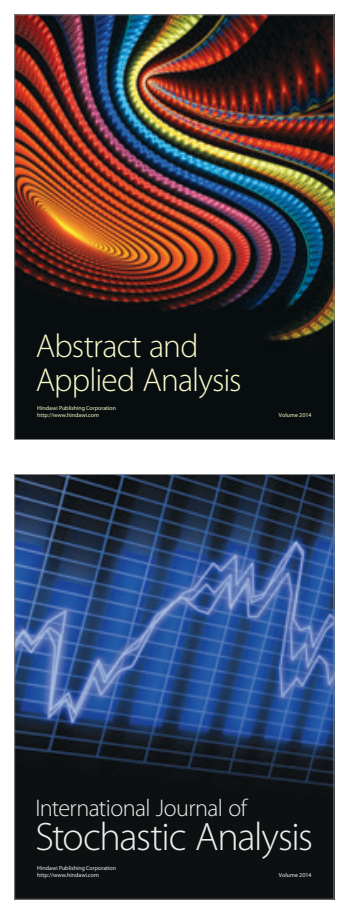

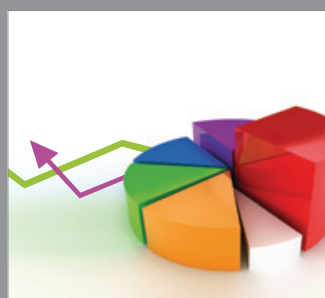

ournal of

Probability and Statistics

Promensencen
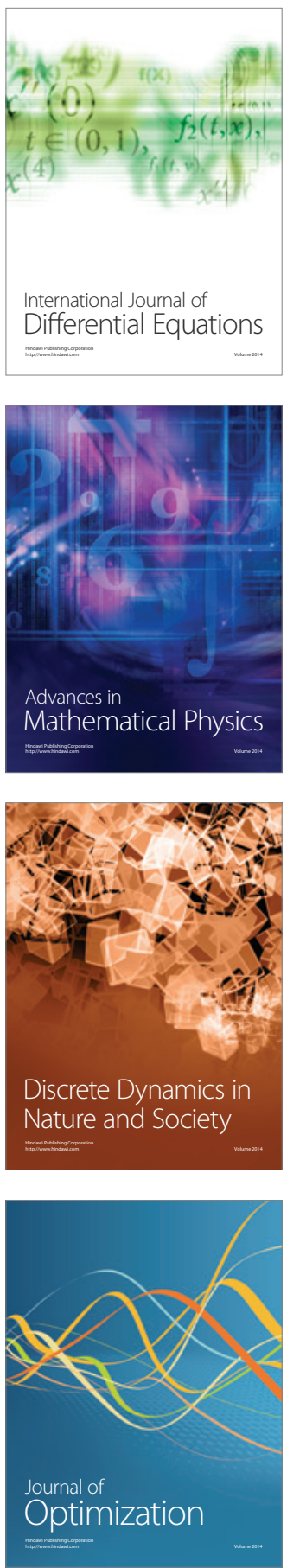